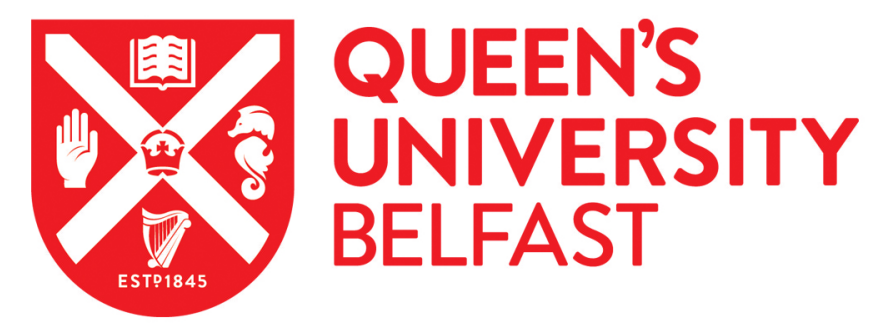

\title{
Design and Development of a Compact UWB Monopole Antenna with Easily-Controllable Return Loss
}

Nikolaou, S., \& Abbasi, M. A. B. (2017). Design and Development of a Compact UWB Monopole Antenna with Easily-Controllable Return Loss. IEEE Transactions on Antennas and Propagation, 65(4), 2063-2067.

https://doi.org/10.1109/TAP.2017.2670322

\section{Published in:}

IEEE Transactions on Antennas and Propagation

\section{Document Version:}

Peer reviewed version

Queen's University Belfast - Research Portal:

Link to publication record in Queen's University Belfast Research Portal

Publisher rights

(c) 2017 IEEE This work is made available online in accordance with the publisher's policies. Please refer to any applicable terms of use of the publisher.

\section{General rights}

Copyright for the publications made accessible via the Queen's University Belfast Research Portal is retained by the author(s) and / or other copyright owners and it is a condition of accessing these publications that users recognise and abide by the legal requirements associated with these rights.

Take down policy

The Research Portal is Queen's institutional repository that provides access to Queen's research output. Every effort has been made to ensure that content in the Research Portal does not infringe any person's rights, or applicable UK laws. If you discover content in the Research Portal that you believe breaches copyright or violates any law, please contact openaccess@qub.ac.uk. 


\section{Design and Development of a Compact UWB Monopole Antenna with Easily-Controllable Return Loss}

\author{
Symeon Nikolaou, and M. Ali Babar Abbasi
}

\begin{abstract}
This communication presents a novel cactus-shaped Ultra Wideband (UWB) monopole antenna fabricated on Liquid Crystal Polymer (LCP). The proposed antenna is a very compact design since it can be fabricated on a board with dimensions only $20 \times 28 \mathbf{m m}^{2}$, while the three linear segments that comprise the cactus-shaped monopole provide a direct control on antenna matching. The proposed antenna is operating from $2.85 \mathrm{GHz}$ to 11.85 $\mathrm{GHz}$ and it presents very consistent omni-directional patterns throughout the UWB frequency range. Return loss and pattern measurements are presented and the operation principles are discussed in detail. The simplicity of this topology, with the easily controllable return loss, allows for its easy implementation for various UWB sub-band designs, just by building suitable monopole versions, for which the only difference is the length of the three linear segments.
\end{abstract}

Index Terms - Cactus antenna, Compact antenna, LCP, U-shaped stub, UWB, UWB monopole

\section{INTRODUCTION}

Since the FCC [1] regulated the 3.1-10.6 GHz band for UWB applications, a significant amount of research activity has been recorded in the area of the design and implementation of UWB antennas. Depending on the application, the requirements for antenna designing may vary significantly, however, for wireless applications, especially for mobile handheld devices, the small size and the omni-directional pattern are highly demanded. For the omni-directional pattern requirements the solution of the printed monopole has been proven very popular and adequately efficient. A circular disk monopole proposed in [2-3] provides consistent omni-directional pattern, however it does not provide any control on the return loss that would potentially allow the operation of the antenna in desired UWB sub-bands (lower and higher). A direct control on the return loss and therefore on the radiated frequencies is achieved by the CPW-fed hexagonal antenna presented in [4] and the composite right/left-handed (DCRLH) transmission line loaded antenna [5], which are used for multi-band applications. CPW-fed monopole and electromagnetic band-gap (EBG) combination antenna that features multi-band and wideband behavior is introduced in [6-7], while CPW-fed monopole with parasitic circular-hat patch is used for the broadband operation in [8] and the L-shaped monopole antenna with hexagonal slot is demonstrated in [9]. However, none of the aforementioned

Manuscript received July 3, 2016. This work was supported in part by the Erasmus Mundus INTACT Doctorate Level Mobility program, funded by the European Union.

Symeon Nikolaou and M. Ali Babar Abbasi are with the Frederick University, Department of Electrical Engineering, 7, Y. Frederickou Str. Pallouriotisa, Nicosia, 1036 Cyprus (phone: +35799218017; e-mail: ali.babar@stud.frederick.ac.cy).

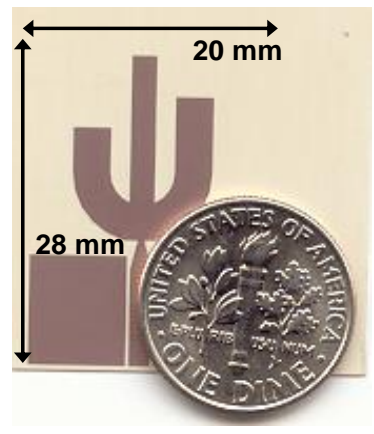

(a)

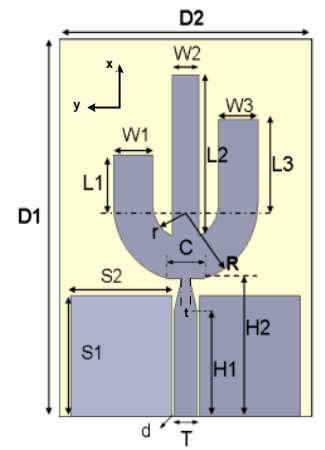

(b)
Fig. 1. Proposed cactus-shaped monopole antenna schematic and fabrication

TABLE I

SCHEMATIC DIMENSIONS

\begin{tabular}{l|llllll}
\hline \multicolumn{7}{|c}{ SCHEMATIC DIMENSIONS } \\
parameter & D1 & D2 & S1 & S2 & H1 & H2 \\
Value $(\mathrm{mm})$ & 28.00 & 20.00 & 9.00 & 8.00 & 7.92 & 10.24 \\
\hline parameter & $\mathrm{L} 1$ & $\mathrm{~L} 2$ & $\mathrm{~L} 3$ & $\mathrm{~W} 1$ & $\mathrm{~W} 2$ & $\mathrm{~W} 3$ \\
Value $(\mathrm{mm})$ & 3.50 & 12.00 & 6.50 & 3.12 & 2.08 & 3.12 \\
\hline parameter & $\mathrm{T}$ & $\mathrm{t}$ & $\mathrm{C}$ & $\mathrm{R}$ & $\mathrm{r}$ & \\
Value $(\mathrm{mm})$ & 1.79 & 0.60 & 2.73 & 5.72 & 2.60 & \\
\hline \hline
\end{tabular}

antennas covers the whole UWB range. Microstrip line fed Fork-shaped [10], U-shaped [11], pentagon-shaped [12] and CPW-fed bowled-shaped [13] planar monopole antennas that cover the whole UWB range have been presented in recent years, however all of these antennas are almost two times the size of the proposed cactus-shaped monopole.

In this letter a compact cactus-shaped monopole UWB antenna is proposed. The presented prototype has board dimensions, $20 \times 28 \mathrm{~mm}^{2}$, is fabricated on low $\varepsilon_{\mathrm{r}}$ flexible organic dielectric material (LCP with $\varepsilon_{\mathrm{r}}=3$ ), presents consistent omni-directional patterns in $H$-plane, and allows direct control on the return loss. The radiation mechanism and the wide band operation are explained in detail, while return loss, pattern and gain measurements are presented to verify its performance.

\section{ANTENNA Design}

The cactus antenna was fabricated on $225 \mu \mathrm{m}$ thick LCP $\left(\varepsilon_{\mathrm{r}}=3, \tan \delta=0.002\right)$ with overall board dimensions $20 \times 28 \mathrm{~mm}^{2}$. The $225 \mu \mathrm{m}$ thick substrate is acquired after two $100 \mu \mathrm{m}$ substrates are stacked and thermo bonded using a $25 \mu \mathrm{m}$ thick LCP bonding layer, having lower melting point, which is sandwiched between the two $100 \mu \mathrm{m}$ thick samples. Standard photolithography is used to print the antenna structure on LCP substrate. The fabricated prototype is presented in Fig. 1(a) and its schematic is shown in Fig. 1(b).

The antenna consists of a CPW line with a linearly tapered broadband transition terminated with a semi-annular ring resonator. Three uneven linear segments are extended from both ends and the center of the semi-annular ring, along the direction of the feed line. Those three different-size linear segments are approximately $\lambda / 4$ long for three intermediate frequencies at which major resonances occur, in the return loss plot (Fig. 2). In a transmission line equivalent circuit, the tapered segment with the cactus-shaped radiator can be 


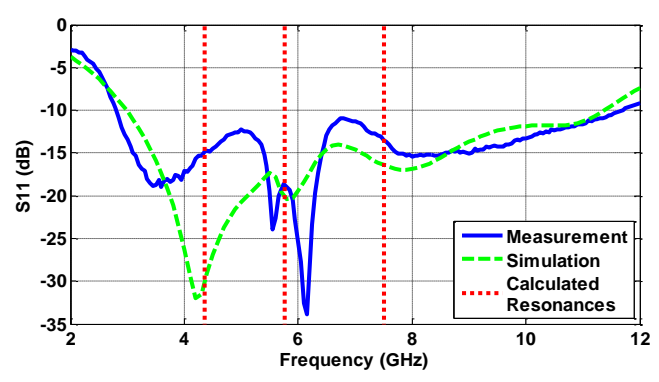

Fig. 2 Measured and simulated return loss. Red dotted line shows the calculated resonance frequency using equation (6).

TABLE II

COMPARISON OF SIMULATED AND CALCULATED RESONANCE FREQUENCIES

\begin{tabular}{l|lll}
\hline \hline Radiator & Calculated $f_{\text {resonance }}$ & Simulated $f_{\text {resonance }}$ & Error \\
\hline Left monopole & $7.56 \mathrm{GHz}$ & $7.85 \mathrm{GHz}$ & $3.6 \%$ \\
Central monopole & $4.37 \mathrm{GHz}$ & $4.20 \mathrm{GHz}$ & $3.8 \%$ \\
Right monopole & $5.75 \mathrm{GHz}$ & $5.90 \mathrm{GHz}$ & $0.9 \%$ \\
\hline \hline
\end{tabular}

considered as a broadband load that terminates a typical CPW line with length $\mathrm{H} 1=7.92 \mathrm{~mm}$. If the equivalent load at the end of the CPW line was equal to the characteristic impedance of the line throughout the whole frequency band of operation, the H1 length would make no difference in the resulted return loss. However, since the impedance of the load changes over frequency, the dimension $\mathrm{H} 1$ is critical in order to achieve good matching. A linearly tapered segment is used as a transition between the CPW line and the radiator. This size of the tapered segment was chosen after a parametric sweep analysis was conducted, in order to achieve the best matching behavior between the CPW line and the ring. From the bottom part of the semi-annular segment, a circular sector is removed resulting in a chord of length $\mathrm{C}=2.73 \mathrm{~mm}$. Since the most important parameter is the length of each stub, the width of the central stub was chosen to be narrower than the width of the side stubs, aiming to increase the distance and therefore reduce the coupling between the neighboring stubs. The overall dimensions of the fabricated antenna are only $20 \times 28 \mathrm{~mm}^{2}$ and the dimensions of schematic are summarized in Table I.

\section{PRINCIPLE OF OPERATION AND RETURN LOSS}

The bandwidth of the circular planar monopoles is larger than any other planar monopole configuration [14]. The main advantage of the circular planar monopole over the rectangular planar monopole can be interpreted in terms of its various resonant modes that are closely spaced, hence supportive for wideband operations like UWB. For a planar 3D monopole, surrounded by air and operating in free space, the lower resonant frequency can be approximately calculated by equating its area $L_{\text {monopole }} \times W_{\text {monopole }}$ to that of an equivalent cylindrical monopole antenna of same height $h_{e q}$, and equivalent radius $r_{e q}$, as described below [14-15]:

$$
r_{e q}=\frac{W_{\text {monopole }}}{2 \pi}
$$

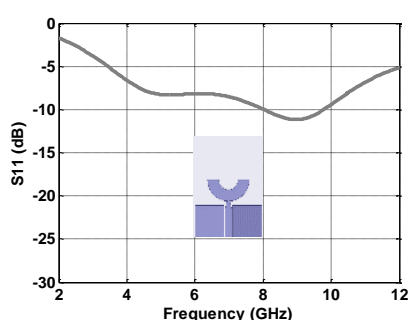

(a)

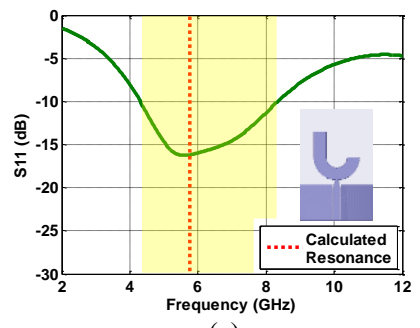

(c)

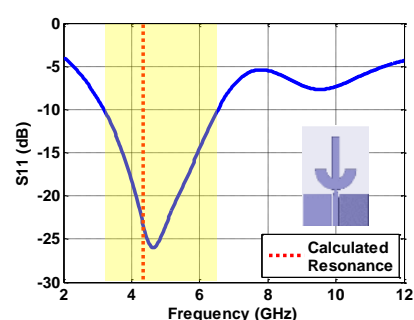

(b)

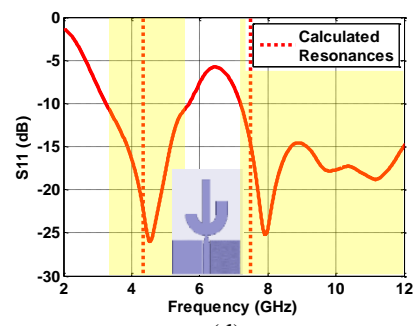

(d)
Fig. 3. Modified versions of the cactus-shaped monopole operating at selected UWB sub-bands when (a) $\mathrm{L} 1=\mathrm{L} 3=0, \mathrm{~L} 2=12$, (b) $\mathrm{L} 1=6.5, \mathrm{~L} 2=\mathrm{L} 3=0$, (c) $\mathrm{L} 1=\mathrm{L} 2=\mathrm{L} 3=0$ and (d) $\mathrm{L} 1=0, \mathrm{~L} 2=11.5, \mathrm{~L} 3=3.5$ (all values in $\mathrm{mm}$ ).

$$
h_{e q}=L_{\text {monopole }}
$$

The input impedance of a $\lambda / 4$ monopole antenna is half of that of the $\lambda / 2$ dipole antenna. Thus, the input impedance of an ideal monopole is $36.5+j 21.25 \Omega$. To make this impedance real, a slightly smaller length of the monopole is used [16]. Where,

$$
L_{\text {monopole }}=0.24 \lambda F
$$

And,

$$
F=h_{e q} /\left(h_{e q}+r_{e q}\right)
$$

Equations (3) and (4) yield:

$$
\lambda=\left(h_{e q}+r_{e q}\right) / 0.24
$$

So, the resonance frequency for a planar monopole can be approximated by:

$$
f=c / \lambda=c \times 0.24 /\left(h_{e q}+r_{e q}\right)
$$

Similar approach is used for the proposed printed on low $\varepsilon_{\mathrm{r}}$ dielectric substrate antenna. Equation (5) does not include the length of the tapered feed, which affects the length of the antenna and consequently the resonant frequency. Accordingly, this equation is modified for the proposed design by incorporating feed length to form:

$$
f_{\text {resonance }}=\frac{c \times 0.24}{h_{e q}+r_{e q}+(H 2-S 1)} H z
$$

Where $c$ is the speed of light in $\mathrm{m} / \mathrm{s}$. The three rectangular segments have been designed based on equation (6). To effectively combine the three linear segments in a single UWB monopole antenna a transition is needed from the CPW feed line. For that scope, a semi-annular ring is used to connect the two side segments. The radius $\mathrm{R}$ of the ring is defined using equation (7) presented in [10] used in slightly different perspective. Instead of the minimum frequency $f_{L}$ a higher, closer to the UWB range center frequency, $f_{R}=5 \mathrm{GHz}$ was used, that allowed the miniaturization of the semi-annular ring and therefore the miniaturization of the proposed UWB cactus-shaped monopole. 


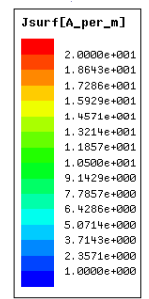

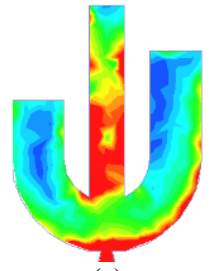

(a)

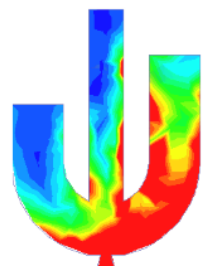

(b)

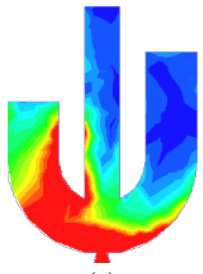

(c)
Fig. 4. Surface current distribution on cactus-shaped radiator at (a) 4.20 $\mathrm{GHz}$, (b) $5.90 \mathrm{GHz}$ and (c) $7.80 \mathrm{GHz}$

$$
f_{R}=\frac{7.2}{2.25 R+(H 2-S 1)} G H z
$$

The design dimensions for the three linear rectangular segments were chosen such as to cover the whole UWB range. The semi-annular ring is used to provide a platform to host these three linear rectangular segments and at the same time provide a smooth and effective transition from the CPW feed line. It has to be noted that the $h_{e q}$ value of the left and right radiators comprise of the combination of $\mathrm{L} 1$ or $\mathrm{L} 3$ respectively, with a portion of the attached semi-annular ring. The effective lengths of left and right segments correspond, and therefore control the highest and intermediate frequencies of UWB spectrum. The calculated values using equation (1) and (2) for the left segment $\left(h_{e q}=9.02 \mathrm{~mm}, r_{e q}=496 \mu \mathrm{m}\right)$ and for the right segment $\left(h_{e q}=12.02 \mathrm{~mm}, r_{e q}=496 \mu \mathrm{m}\right)$ correspond to $7.56 \mathrm{GHz}$ and $5.75 \mathrm{GHz}$, respectively. The effective length of the central segment comprises of a rectangular strip with dimensions $L 2 \times$ $W 2$ and the portion of the semi-annular ring connecting it to the antenna feed line. The central segment length affects the lower frequencies of the return loss (Fig. 2). The calculated values using equation (1) and (2) $\left(h_{e q}=16.13 \mathrm{~mm}, r_{e q}=331 \mu \mathrm{m}\right)$ for the middle segment yields the lowest resonance frequency of the cactus-shaped monopole at $4.37 \mathrm{GHz}$.

All three calculated resonance frequencies are indicated in Fig. 2 along with simulated return loss in comparison with the measured results. The proposed antenna was designed using full wave EM simulators. Three resonances can be observed from the simulated and measured return loss plot. Each resonance mainly depends on the length of the corresponding linear segment. The comparison of the calculated and simulated resonance frequencies for these segments is summarized in Table II. The combined dimensions of the tapered line, ground, semi-annular ring, along with the strategically calculated resonances of each radiating stub, ensure good impedance matching for the antenna throughout the UWB frequency range.

The imminent effect of the lengths L1, L2 and L3 -as implied by equation (6)-, on the resonances of the, thereby, easily controllable return loss, allows the modification of the presented antenna by only changing these three lengths, in order to design antennas that may be used for selected UWB sub-bands. The common platform and three distinct cases, are presented in Fig. 3 where lengths L1, L2 and L3 were chosen such as to match the antennas in different sub-bands. Fig. 3(a) shows the return loss, when all the lengths are set to zero, creating a semi-annular ring monopole which in clearly not well matched. Using this common platform, a lower sub-band

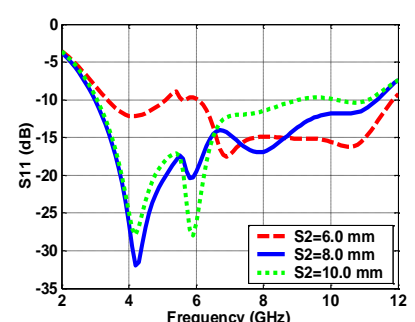

(a)

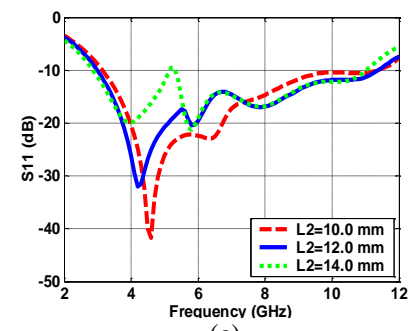

(c)

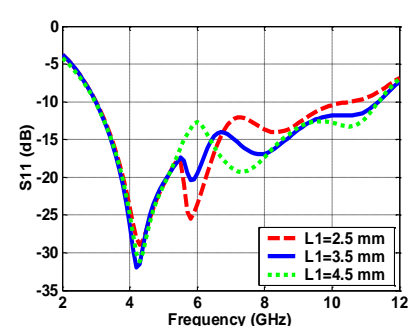

(b)

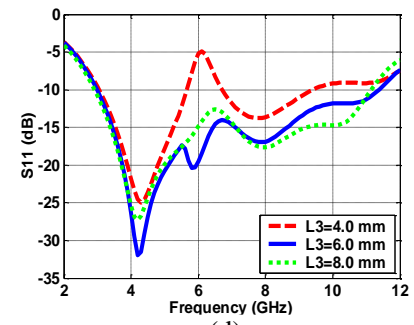

(d)
Fig. 5. Parametric study for the design parameters effect on the return loss. (a) Effect of ground plane (b) left monopole effect (c) middle monopole effect (d) right monopole effect. In each plot other than the varied parameter the other parameters have the values indicated in Table 1.

(Fig. 3(b)), a middle sub-band (Fig. 3(c)), and a UWB antenna with a band-stop frequency notch, (Fig. 3(d)) can be designed. In all three cases only lengths L1, L2 and L3 are changed and every other design dimension remains the same, as summarized in Table I. As can be observed in Fig. 3, the predicted resonances from equation (6) (dotted lines) that fall within the UWB range are in good agreement with the simulated resonant frequencies.

The surface current distribution on the antenna is presented in Fig. 4. where the higher tone (red in online colored version) represents the strongest surface current. It is obvious from the surface current plot at $7.80 \mathrm{GHz}$, that most of the power is radiated from the left stub of the antenna, while at $5.90 \mathrm{GHz}$ the right stub radiates the maximum power. The longest linear segment in the middle of the antenna is most critical for the radiation at the lowest resonance frequency centered at 4.20 $\mathrm{GHz}$.

The effect of the ground patch length and the effect of each of the three linear segments' length, were parametrically studied, and the results are compiled in Fig. 5. The contour with the solid line in all four plots in Fig. 5. presents the optimized design's simulation results. The width of the ground plane, S2 directly affects the impedance matching at the lower frequencies, when S2 $=6 \mathrm{~mm}$. Similar mismatch is observed at higher frequencies when $\mathrm{S} 2=10 \mathrm{~mm}$. The optimized value of $\mathrm{S} 2$ is $8 \mathrm{~mm}$, for which the antenna is matched everywhere in the UWB band, as can be seen in Fig. 5(a). Figs 5(b), (c) and (d) reveal the impact of each stub's length on the position of the respective resonance. In general, the increase in the length of a stub causes the related resonance to shift towards lower frequencies. This tendency, remains consistent as can be seen in every one of the three plots mentioned above. The same behavior has been theoretically predicted from equation (6). One of the most common issues in the resonances distribution approach, for wide band antenna designs, is the 'collapse of resonance'. This collapse often reveals a peak in the return loss 

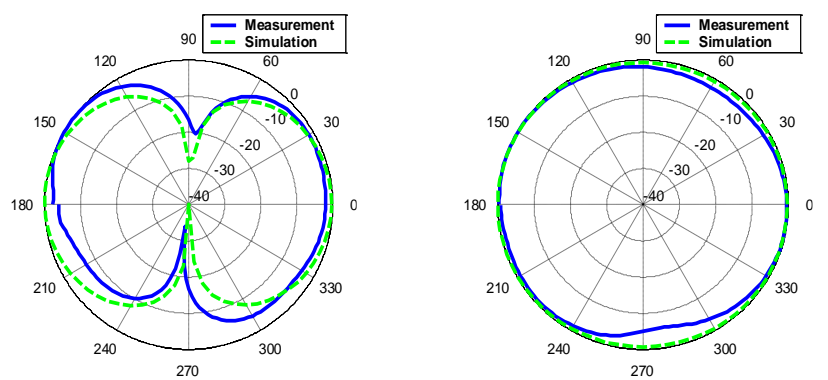

(a)

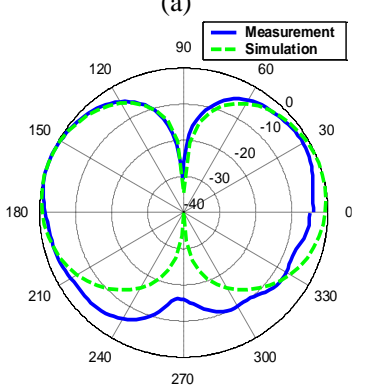

(c)

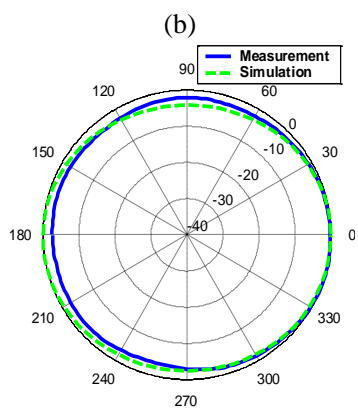

(d)

Fig. 6. Comparison between simulated and measured normalized radiation patterns (a) $E$-plane (b) $H$-plane at $4 \mathrm{GHz}$, (c) $E$-plane and (d) $H$-plane at 8 $\mathrm{GHz}$

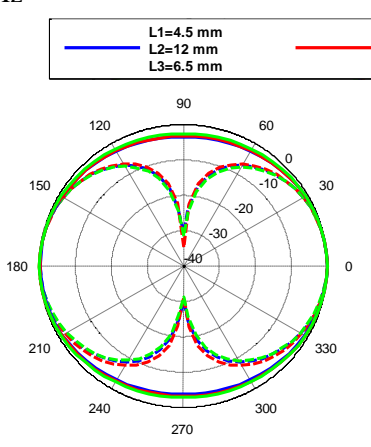

(a)

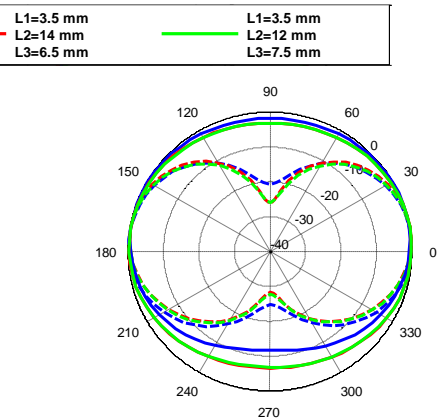

(b)

Fig. 7. Simulated antenna radiation patterns for three different variations of the lengths of monopoles where dotted and solid contours show the $E$-plane and $H$-plane cuts respectively at (a) $4 \mathrm{GHz}$ and (b) $8 \mathrm{GHz}$.

plot at the frequency band where the resonance previously existed. This phenomenon can be observed in Fig. 5(b) for L1 = $4.5 \mathrm{~mm}$. A similar mismatch problem is often observed when the two resonances occur too far apart from each other. Such case can be witnessed in Fig. 5(c) for L2=14 $\mathrm{mm}$ in which the first resonance shifts to lower frequencies, resulting in a peak of return loss between the first two resonances. The most intense mismatch peak can be observed from Fig. 5(d) when L3 $=4$ $\mathrm{mm}$. The reason behind this peak is the position of the second resonance far away from the first resonance and its merge with the third resonance, eventually resulting in significant mismatching of the $\left|S_{11}\right|$ around $6 \mathrm{GHz}$. The size of the ground plane (S2) is an important parameter for the overall matching of the antenna as it affects the position as well as the depth of each resonance. On the other hand, the length of each stub does not significantly affect the depth or the position of the resonances created by the other two stubs. It is evident that while a certain stub only affects its corresponding resonance, the ground patches and the cactus-shaped radiator should be considered as a single object, in order to achieve good matching results

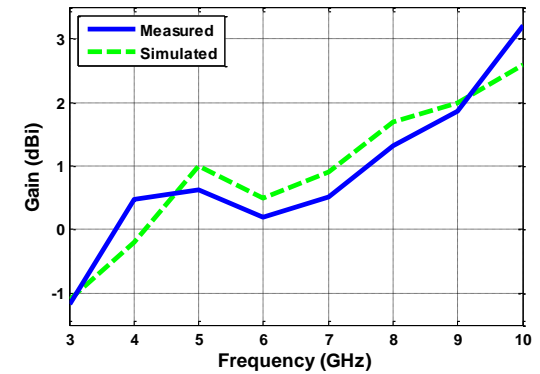

Fig. 8 Measured and simulated gain in $\mathrm{z}$ axis direction

TABLE III

COMPARISON BETWEEN REPORTED PLANAR MONOPOLE TYPE ANTENNAS

\begin{tabular}{llllll}
\hline \hline Ref. & Dimensions & Freq. range & \multicolumn{2}{l}{ Peak gain $(\mathrm{dBi})$} & \multicolumn{2}{l}{ Size } \\
& $(\mathrm{mm})$ & $(\mathrm{GHz})$ & range & avg. & comp. $(\%)$ \\
\hline$[2-3]$ & $50 \times 50$ & $2.60-10.20$ & $-2.6-6.0$ & 2.27 & 446.4 \\
{$[13]$} & $44 \times 38$ & $3.05-12+$ & - & - & 298.6 \\
{$[11]$} & $40 \times 30$ & $2.26-22.18$ & $3.3-6.8$ & 4.53 & 214.3 \\
{$[10]$} & $42 \times 24$ & $2.30-2.50$ & $1.0-5.4$ & 3.68 & 180.0 \\
{$[8]$} & $30 \times 30$ & $3.10-12.00$ & $2.7-5.3$ & 4.12 & 160.7 \\
& & $3.40-7.62$ & 2.7 & \\
{$[4]$} & $25 \times 26$ & $3.30-3.66$ & $1.6-2.2$ & 1.97 & 116.1 \\
& & $5.10-6.00$ & & & \\
{$[5]$} & $26.2 \times 18$ & $3.06-3.43$ & $2.2-3.6$ & 2.67 & 84.1 \\
This & $28 \times 20$ & $4.55-5.86$ & & & \\
work & $2.85-11.85$ & $-1.2-3.2$ & 0.84 & 100.0 \\
\hline
\end{tabular}

throughout the complete UWB frequency range.

\section{RADIATION PATTERN AND GAIN MEASUREMENTS}

Fig. 6 presents the comparison of simulated and measured normalized radiation patterns in both $E$ and $H$-planes at 4 and 8 GHz. Based on the antenna orientation described in Fig. 1(b) $\mathrm{x}-\mathrm{z}$ and y-z planes represent $E$ and $H$-planes respectively. It is evident from the radiation patterns that the antenna is exhibiting, close to typical monopole antenna radiation patterns, in both frequencies, and in both planes, as well. The plots also confirm the sustainability in radiation performance of the cactus-shaped antenna throughout the UWB frequency range. Measured radiation patterns are in good agreement with the simulated predictions, validating the performance of the fabricated prototype. Although the antenna is not symmetrical, the presented radiation patterns are almost symmetrical. The expected asymmetry in the radiation patterns should be reflected on $x-y$ plane cuts, however the measured patterns are presented only in $\mathrm{x}-\mathrm{z}$ and $\mathrm{y}-\mathrm{z}$ planes. Fig. 7 implies that the primary parameters that control the return loss (i,e, L1, L2 and L3) have minor effect on the antenna's radiation patterns which remain consistently omnidirectional along the $H$-plane.

The gain of the antenna is measured using the substitution method, in which a 2 to $18 \mathrm{GHz}$ Q-par Angus Ltd rectangular horn antenna of known gain was used. The gain accuracy of the horn antenna is within $\pm 0.8 \mathrm{~dB}$ range, thus this can be considered as the accuracy of the measurement setup for the cactus-shaped antenna. A direct comparison of simulated and measured gain in complete UWB frequency range is presented in Fig. 8. Maximum gain is measured in $\varphi=\theta=0^{\circ}$ i.e. in the 
direction of z-axis and the agreement between measurements and simulated results is evident. The gain of the antenna grows linearly with respect to frequency and the overall gain remains within $4 \mathrm{~dB}$ range. The very consistent omnidirectional radiation patterns of the antenna, in $H$-plane, is the reason that maximum gain is kept low as can be deduced from Table III that provides a comparison between this work and the similar antennas reported in the references. The consistent omni-directional patters in combination with the compact size of the cactus-shaped monopole make the proposed antenna a good candidate for most UWB applications.

\section{CONCLUSION}

A novel CPW fed, compact, cactus-shaped monopole antenna fabricated on low loss, flexible, $225 \mu \mathrm{m}$ thick LCP organic material is presented. The radiation mechanism of the antenna, depends on three radiating stubs, connected to a semi-annular ring creating a cactus shape radiator. The resonances of the antenna were initially predicted with an analytical formula using classical theory of CPW-line-fed, semi-annular, and rectangular, radiators. Full wave EM simulator was further used to optimize the performance of the antenna to exhibit good impedance matching throughout the whole UWB frequency range. The lengths of the three radiating stubs control the position and depth of $\left|S_{11}\right|$ resonances, hence allow a direct control on the return loss and therefore on the antenna matching. This feature allows a readjustment of the antenna characteristics to focus on different UWB sub-bands, making the presented antenna a potential candidate for next generation UWB transceivers, which may operate in designated sub-bands, depending upon the specifications of the desired application.

\section{ACKNOWLEDGMENT}

The authors would like to thank colleagues from GaTech Athena and MircTech research groups, and from NASA Glenn for their contribution with testing.

\section{REFERENCES}

[1] "Federal Communications Commission revision of Part 15 of the commission's rules regarding ultra-wideband transmission systems," FCC, Washington, DC, First Report and Order FCC, 02.V48, 2002.

[2] J. Y. Siddiqui, C. Saha, Y. M. M. Antar, "Compact Dual-SRR-Loaded UWB Monopole Antenna With Dual Frequency and Wideband Notch Characteristics," IEEE Antennas and Wireless Propagation Letters, vol.14, pp. 100-103, 2015.

[3] J. Y. Siddiqui, C. Saha and Y. M. M. Antar, "Compact SRR Loaded UWB Circular Monopole Antenna With Frequency Notch Characteristics," in IEEE Transactions on Antennas and Propagation, vol. 62, no. 8, pp. 4015-4020, Aug. 2014.

[4] Wu Rui-Zhi, Wang Peng, Zheng Qiang, Li Rui-Peng, "Compact CPW-fed triple-band antenna for diversity applications," Electronics Letters, vol. 51, no. 10, pp. 735-736, 2015.

[5] Li Hai-Peng, Wang Guang-Ming, Gao Xiang-Jun, Zhu Li, "CPW-Fed Multiband Monopole Antenna Loaded With DCRLH-TL Unit Cell," IEEE Antennas and Wireless Propagation Letters, vol. 14, pp. 1243-1246, 2015.

[6] M. E. de Cos, F. Las-Heras, "Dual-Band Uniplanar CPW-Fed Monopole/EBG Combination with Bandwidth Enhancement," IEEE Antennas and Wireless Propagation Letters, vol.11, pp. 365-368, 2012.
[7] M. E de Cos, F. Las-Heras, "Polypropylene-Based Dual-Band CPW-Fed Monopole Antenna [Antenna Applications Corner]," IEEE Antennas and Propagation Magazine, vol. 55, no. 3, pp. 264-273, June 2013.

[8] Liu Wen-Chung, Wu Chao-Ming, Tseng Yen-Jui, "Parasitically Loaded CPW-Fed Monopole Antenna for Broadband Operation," IEEE Transactions on Antennas and Propagation, vol. 59, no. 6, pp. 2415-2419, June 2011.

[9] Zhou Shui-Wei, Li Ping-Hui, Wang Yang, Feng Wei-Hua, Liu Zong-Quan, "A CPW-Fed Broadband Circularly Polarized Regular-Hexagonal Slot Antenna With L-Shape Monopole," IEEE Antennas and Wireless Propagation Letters, vol. 10, pp. 1182-1185, 2011.

[10] S. K. Mishra, R. K. Gupta, A. Vaidya, J. Mukherjee, "A Compact Dual-Band Fork-Shaped Monopole Antenna for Bluetooth and UWB Applications," IEEE Antennas and Wireless Propagation Letters, vol. 10, no. , pp. 627-630, 2011.

[11] Tang Ming-Chun, R. W. Ziolkowski, Xiao Shaoqiu, "Compact Hyper-Band Printed Slot Antenna With Stable Radiation Properties," IEEE Transactions on Antennas and Propagation, vol. 62, no. 6, pp. 2962-2969, June 2014.

[12] R. A. Moody, S. K. Sharma, "Ultrawide Bandwidth (UWB) Planar Monopole Antenna Backed by Novel Pyramidal-Shaped Cavity Providing Directional Radiation Patterns," IEEE Antennas and Wireless Propagation Letters, vol. 10, pp. 1469-1472, 2011.

[13] M. Koohestani, N. Pires, A. K. Skrivervik, A. A. Moreira, "Influence of the human body on a new coplanar-fed Ultra-Wideband antenna," 6th European Conference on Antennas and Propagation (EUCAP), pp. 316-319, 26-30 March 2012.

[14] G. Kumar and K. P. Ray, Broadband Microstrip Antennas, Norwood, MA- Artech house, 2003.

[15] R. Garg, et al., Microstrip Antenna Design Handbook, Norwood, MA: Artech House, 2001.

[16] C. A. Balanis, Antenna Theory Analysis and Design, New York: John Wiley \& Sons, 1997. 